\begin{tabular}{l}
\hline Letters may be edited for space and clarity. \\
They should be addressed to: \\
Correspondence \\
Nature Biotechnology \\
345 Park Avenue South \\
New York, NY 10010-1707, USA \\
or sent by e-mail to biotech@natureny.com \\
Please include your telephone and fax numbers.
\end{tabular}

More views of Cartagena

To the editor:

In "Four views from Cartagena" (Nature Biotechnology 17, 512, 1999), the first piece, "New confrontations" by Elizabeth Hodsonde-Jaramillo and Rafael Aramendis starts by stating that "Article 19.3 of the Convention

Biological Diversity establishes the need to create a legally compelling instrument...

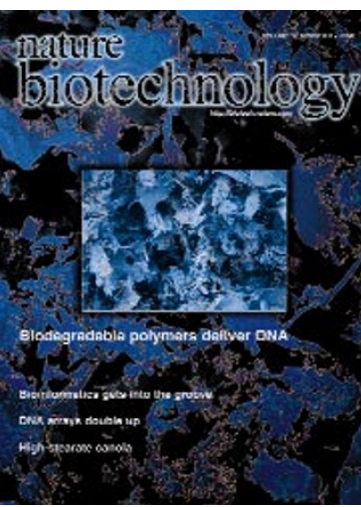

we look to you as a journal of authoritative record to be scrupulous in such matters.

Mark Cantley

Brussels, Belgium mark.cantley@dgiz.cec.be

1. http://www.biodiv.org/

To the editor:

Recent commentaries on the Biosafety Protocol negotiations (Nature Biotechnology, $17,512,1999)$ analyzed various reasons for suspension of the final meeting held in Cartagena, Colombia, in February of this year. From a scientific point of view, we would like to emphasize that the draft protocol ${ }^{1}$ contains innovative elements with respect to the safe handling, transfer and use of living modified organisms (LMOs).

In an attempt to implement the precautionary approach, lack of scientific knowledge or scientific consensus should not necessarily be interpreted as indicating a particular level of risk, an absence of risk, or an acceptable to regulate the transfer, handling and use of "living modified organisms produced by modern biotechnology that could have an adverse effect. ..."

Sorry, it doesn't. The text is available on the excellent website of the CBD Secretariat ${ }^{1}$, and Article 19.3 states, in full: "The Parties shall consider the need for and modalities of a protocol setting out appropriate procedures, including, in particular, advance informed agreement, in the field of the safe transfer, handling and use of any living modified organism resulting from biotechnology that may have adverse effect on the conservation and sustainable use of biological diversity."

Following the Rio Summit of June 1992, the executive director of UNEP (UN Environment Program) charged four expert groups with reporting on how to implement the convention-one group being specifically charged to report on Article 19.3. As per the official text, we "considered the need for" a protocol, and opinion was divided; also as to appropriate modality (binding or advisory) if protocol there had to be. I was among those unconvinced of the need, and our chair, Veit Koester (subsequently chair of the Biosafety Working Group drafting a protocol), with the fair-mindedness for which he is renowned, agreed to our publishing (in 1993) a split report, recording both the case for a binding protocol, and the counter-arguments of those who saw neither scientific nor political need, and many practical reasons against.

Given the protracted time-scales typical of international negotiations, it is important that history be not rewritten, nor the original charge inadvertently (or otherwise) revised; risk. In addition, where there is uncertainty regarding the level of risk, the annex on risk assessment calls for appropriate risk management strategies and complementary monitoring.

The concept of monitoring is to verify assumptions made in a risk assessment and to evaluate whether risk management measures are appropriate and effective ${ }^{2}$. Crop- and traitspecific monitoring programs for transgenic plants already exist in the United States and Canada and are currently being elaborated in the European Union.

Thus, monitoring potential adverse effects on biological diversity in the receiving environment can contribute significantly to gaining experience with the application of LMOs. When introducing transgenic plants on a global scale, therefore-with a Biosafety Protocol in place-both environmental risks and collateral economic losses could be controlled.

Bernhard Jank ${ }^{1}$, Johannes Rath ${ }^{2}$ and Helmut Gaugitsch ${ }^{3}$ ${ }^{1}$ Institute of Applied Microbiology, University of Agriculture, Nussdorfer Lände 11, A-1190 Vienna, Austria ${ }^{2}$ Institute of Zoology

University of Vienna, Althanstrasse 14 A-1090 Vienna, Austria ${ }^{3}$ Federal Environment Agency Spittelauer Lände 5, A-1090 Vienna, Austria Gaugitsch@ubavie.gv.at

1. UNEP/CBD/BSWG/6/L.2/Rev.2 (1999) Protocol on Biosafety. Draft text submitted by the chair of the working group, Open-ended ad hoc working group on Biosafety, Cartagena, Colombia.

2. UNEP (1995) International Technical Guidelines on Safety in Biotechnology, United Nations Environment Program, Nairobi, Kenya.

\section{Cloning nonuniformity}

To the editor:

While reading about developmental defects and high rates of mortality among animals produced by cloning (Nature Biotechnology 17, 405, 1999), we were reminded of similar problems encountered by plant scientists when cloning plants from cultured somatic cells. Considerable phenotypic variability has been observed among presumably genetically identical plants regenerated from cell or tissue culture, a phenomenon that has been called somaclonal variation ${ }^{1}$. Although the basis of somaclonal variation remains unclear, a substantial epigenetic component has been proposed ${ }^{2,3}$. In particular, developmentally acquired epigenetic states, which would normally be reset during sexual reproduction, can be stably maintained during regeneration of plants from somatic cells, resulting in the silencing of genes that normally would be expressed ${ }^{3}$.

Some of the abnormalities and deaths of cloned mammals might relate to disruptions in the epigenetic phenomenon of parental imprinting. However, imprinting disturbances probably do not account for all of the problems in cloning ${ }^{4}$, possibly implicating more general epigenetic alterations involving nonimprinted genes. Whatever proves to be the source of abnormalities in cloned animals, a lesson from plants is that "clonal uniformity is the exception rather than the rule"5. M.A. Matzke and A.J.M. Matzke Institute of Molecular Biology Austrian Academy of Sciences Billrothstrasse 11 A-5020 Salzburg, Austria mmatzke@imb.oeaw.ac.at

1. Larkin, P.J. et al. Theor.Appl. Genet. 60, 197-214 (1981).

2. Kaeppler, S.M. et al. Proc. Natl. Acad. Sci. USA 90, 8773-8776 (1993)

3. Matzke, M.A. et al. In Epigenetic Mechanisms of Gene Regulation (eds Russo, E., Martienssen, R. \& Riggs, A.) 377-392 Cold Spring Harbor Press, New York, (1996).

4. Weiss, R. Washington Post, May 10, 1999

5. Scowcroft, W.R. In Genetic flux in plants (eds Springer-Verlag, Vienna; 1985).

\section{Next month in nature biotechnology:}

Fermenters and bioreactors High-throughput screening and assays 